\title{
Stimulating Team Creativity: The Influence Of Swift-Trust On The Team Creativity Process
}

Dr. John Berthold, D.M., Sunhillo Technical Services Corporation, USA

\begin{abstract}
It is often suggested that most creative ideas that lead to an innovation originate from a collaborative environment. Organizations frequently establish teams to encourage collaboration as a way of generating new and creative ideas for innovation. Yet, teams often fail to establish a proper collaborative environment in a timely manner or to stimulate team creativity. Team creativity, a by-product of the proper collaborative experience, and its stimulating agents are the focus of this paper. Specifically, this paper examines how organizations can rapidly create the proper collaborative environment to induce team creativity using swift-trust. Swift-trust, a unique form of trust and collective perception, is proposed as a stimulating agent for the quick formation of a collaborative environment that leads to team creativity. The antecedents to the formation of swift trust are identified as team composition and team tasking. The moderating effects of the leader-member relationship and history are examined. Overall, results show that swift trust leads to the rapid establishment of a collaborative team environment which leads to team creativity. A conceptual model is presented and an example is discussed. This paper makes two important contributions. First, the establishment of a swift-trust environment is positively linked to the early stimulation of team creativity. Second, a practical blueprint, through the development of a conceptual model, is offered to the management practitioner desiring to quickly stimulate team creativity using swift-trust.
\end{abstract}

Keywords: Creativity; Innovation; Trust; Swift Trust; Team Creativity

\section{INTRODUCTION}

¿ n today's competitive environment, companies cannot afford to waste time or remain static. Innovation, or the introduction and implementation of new ideas, processes, or products (West \& Farr, 1990), are central to an organization's ability to stay competitive. Additionally, the pace of change appears to be accelerating, compelling companies to rapidly provide new innovations in order to compete and to survive (Amabile, 1997). Creativity is the first step in innovation. According to Amabile (1996) creativity is defined as "the production of novel and appropriate ideas found in any domain" (p. 1). And, it is creativity, the antecedent of innovation, which is required in order for innovation to take place. The ability of any company to stimulate creativity quickly is crucial to its long-term survival.

While scholarly discussions on creativity are abundant, practical prescriptions on how to rapidly stimulate creativity, especially within the team environment, are lacking. Creativity is typically portrayed as an individual construct and as a collaborative process. Literature on individual creativity emphasizes the pro-active motivational state or pro-active personality of the individual, while team creativity is generally discussed as an outcome of transformational leadership (Chen et al., 2013). The transformational leader drives individual creativity by motivating individuals, boosting morale, and urging goal accomplishments. In the practical world, however, it is recognized that most ideas which lead to innovation stem from the collaborative environment, where groups of people work together to solve complex tasks (Barczak et al., 2010). It is within this context, the proper collaborative environment, where individuals "talent, energy and skills are integrated into a team, and this collective capacity to 
innovate becomes greater than the sum of individual contributions" (Chen, 2007, p.2). Put simply, creativity is an essential element in team success, and it is creativity, the forerunner to innovation, which enables teams to solve problems to produce innovation. So, how can organizations quickly establish the right collaborative environment that stimulates team creativity? One answer is through "trust", specifically, with the concept referred to as "swifttrust".

Swift-trust refers to a type of trust that is established quickly in teams, where a unique form of collective perception develops, that displaces any individual anxieties of vulnerability, uncertainty, risk and expectations in order to stimulate the collective accomplishment of group goals (Meyerson et al., 1996). In the swift-trust environment, team members are freed from the usual inhibitions, such as the willingness to trust others, that sometimes impede the creative thought process. As such, swift-trust can quickly lead to the establishment of a collaborative environment that encourages team creativity and inspires team members to take action. It is the linkage between swift-trust and a collaborative environment that is conducive to team creativity that has practical implications.

It is a central premise of this paper that the creation of swift trust leads to a collaborative team environment which leads to team creativity. The remainder of this paper is dedicated to answering several questions: how does swift-trust develop and rapidly lead to a collaborative environment which leads to team creativity? Secondly, what moderating factors affect team creativity? In answering these questions, this paper reviews literature pertaining to team creativity, swift-trust and its antecedents which lead to a proper collaborative team environment for team creativity to thrive. The moderating influences of leader-member relationships and history between members are discussed. Further, a conceptual model of these relationships will be presented. This will be followed by a discussion, management implications, limitations and future research potentials.

\section{TEAM CREATIVITY}

Creativity, in general, is the production of novel and appropriate ideas, regardless of the domain is which they are created (Amabile, 1996). And, team creativity occurs when team members work in integrated ways to capitalize on their diverse knowledge and skills (West, 2002) to produce novel and useful ideas for innovation.

Team creativity is an outcome of a positive collaborative environment. A number of scholars have examined team creativity from an organizational perspective (King \& Anderson, 1990), while others look upon team creativity as a function of individual characteristics (Agrell \& Gustafon, 1996). Paulus (2000) postulates that high team creativity, or its opposing proposition of low team creativity, is an outcome of the social and cognitive stimulations provided by its members. Amabile $(1988 ; 1996)$ assumes individual and team-level creative processes to be of similar composition since both involve cognitive processes of idea generation and idea testing. Team creativity is also strongly associated with the morale and climate established for the team (Chen et al., 2013). However, at its core, team creativity is strongly influenced by the collaborative environment that is established. The proper collaborative team environment will inspire individuals to work together in order to produce ideas that are both novel and useful to the organization.

\section{COLLABORATIVE TEAM ENVIRONMENT}

The concept of the team dynamic, collaboration, and the need for cooperative systems within organizational structures in order to accomplish tasks are not new to the study of management. Barnard (1958) was one of the earliest modern organizational theorists to recognize the importance of cooperative systems within organizations. As posited by Barnard, "an essential element of organizations is the willingness of persons to contribute their individual efforts to the cooperative system" (Barnard, 1958, p. 139). Schein (2004) posits that the strength and stability of organizational culture derives from the fact that it is group based. Kramer (2010) theorizes that the collective entity is unique because its purpose is to service the organization's needs as a whole, instead of individual interests. Within this context, the use of teams as cooperative systems to generate new ideas is a natural extension. 
Organizations often use temporary teams as a way to generate new ideas. Temporary teams are formed around a particular set of objectives, goals or purposes. The temporary environment provides "a set of diversely skilled people working together on complex tasks over a limited period of time" (Goodman \& Goodman, 1976, p.494). Examples of these temporary teams might be Research and Development (R\&D) teams, "tiger" teams organized to solve specific issues, cockpit crews or theater production crews. The use of temporary teams reduces the problems that are sometimes seen with long-term team associations, such as a narrowing of thinking, reduction in quality output, or the reduction of idea generation (Kurtzberg \& Amabile, 2001). The temporary environment is important because it provides a set of unique characteristics that fosters creativity and self-reliance amongst its team members. Included amongst the general prerequisites for this environment are task assignments which are complex, non-routine, time sensitive and carry importance to the organization. However, the key binding element that makes this team environment successful is "trust". Without the element of trust, collaborative teams may not be successful and fail to generate new ideas or to stimulate team creativity.

Trust improves team creativity (Goodman \& Goodman, 1976) and is often referred to as the "social glue" which binds teams together in order to accomplish important goals. Trust denotes the willingness of one party to take on the risk of being vulnerable to the action of another party (Kramer, 1999). Within the collaborative team environment as described above, member behaviors presupposes trust because the traditional sources of trust such as familiarity, shared experience, reciprocal disclosure and fulfilled promises are not present. The development of trust and its maturity can take time (Kramer, 1999) - precious time, which organizations and teams value and cannot afford to waste. So, how can organizations quickly establish the right collaborative environment that stimulates team creativity? To propose an answer to this question, this paper looks at the concept of swift trust.

\section{SWIFT-TRUST}

Swift-trust can be used to quickly establish an environment of trust within teams. Further, swift-trust promotes team creativity by establishing a collaborative team dynamic that encourages both individual and group creativity to thrive.

Meyerson et al. (1996), in a seminal work on the origins and theory of swift-trust, imply a direct connection between the creation of a swift-trust environment, team accomplishments and creativity. As defined, swift-trust refers to a unique form of collective perception which often develops within temporary teams (e.g., temporary systems environments). Swift-trust is an enabler in rapidly establishing team trust and in getting work done. It displaces the usual individual anxieties of vulnerability, uncertainty, and risk that are associated with normal trust development in favor of a collective desire to accomplish team goals. As the authors aptly state, swift-trust "is less about relating than doing" (p. 191). The connection between swift-trust and the environment necessary for team creativity is provided by reference to the forerunner studies of Goodman and Goodman (1972 \& 1976).

Swift-trust produces an environment conducive to team creativity. Goodman and Goodman (1972 \& 1976) study the team dynamics of theater production crews during various theater performances that take place throughout California. The authors are curious as to why some theater productions fail early in the theater season while others seem to quickly adapt and succeed for the long season. The authors theorize the issue is somehow related to team dynamics, which warrants these studies. From the studies, the authors conclude that there are two types of production teams operating: (a) those production teams with long prior histories of working together, where roles and duties are very clear - these types of crews are able to technically produce a play but, as observed, they lack the creativity to change or to offer new ideas on how to improve the plays; and, (b) there are production crews that have little prior history and there is a blurring of specific roles - in these types of teams, members adapt quickly to prevent potential failures by pulling together to suggest new ideas going forward (i.e., creativity) that help the play to succeed. These studies are important because they provide a clear linkage between the establishment of a swift trust environment and team creativity.

A number of scholars have expanded upon these earlier studies by suggesting the use of swift trust as a stimulating agent for the rapid formation of trust in teams. Robert, Dennis and Hung (2009) adopt swift trust and apply its concepts into a model of trust formation, suggesting swift trust is a critical component in lifecycle of trust development. Jarvenpaa et al. (1998) connect swift trust with the establishment of high trust levels seen in teams. 
From these and earlier studies, a clearer picture of the antecedents necessary for the formation of swift trust can be derived.

\section{ANTECEDENTS TO THE FORMATION OF SWIFT TRUST}

Swift trust requires the establishment of a temporary systems environment (Meyerson et al., 1996). To accomplish this, team composition and team tasking have particular relevance to the encouragement of collaboration and interdependence (Goodman \& Goodman, 1972 \& 1976; Meyerson et al., 1996). To convert the individual expertise of strangers into an interdependent working environment means people must suppress their normal anxieties about each another. This requires the careful creation of a team and associated tasking which encourages collaboration, interdependence and idea generation.

\section{TEAM COMPOSITION}

There are four primary team composition factors which help to promote the creation of swift trust in teams: (a) a diverse pool of talent; (b) work histories; (c) limited prospects of working together again; and, (d) a limited team size (Goodman \& Goodman, 1972 \& 1976; Meyerson et al., 1996).

Diverse pool of talent. Team members with a diverse set of skills and expertise offer a greater collective knowledge and are more likely to work across normal functional domains in order to get goals accomplished. Expectations for participation in the team are defined more in terms of tasks and capabilities than specialty domains. This encourages interdependence when required to get the job done.

Limited working histories. In the temporary systems environment, members have little time to sort out who knows precisely what. As such, members with limited histories of working together exhibit behavior that presupposes trust and expectations that other members are trustworthy. This encourages immediate team participation.

Limited prospects of working together again. Members with limited prospects of working together again will have little fear of repercussions or reprisals for their current actions. If members perceive there is a limited possibility of a diffusion of information about the project outcomes or member performances, then there is also limited reputational risk associated with current activity. This encourages immediate participation in team activities and drives collaborative behavior.

Limited team size. The smaller the team size, the more likely individuals are to trust in each other, collaborate, and depend on each other in order to complete expected goals.

\section{TEAM TASKING}

There are four primary steps recommended regarding team tasking: (a) tasks should be complex; (b) tasks should be non-routing; (c) tasks should be time sensitive; and, (d) task should have an importance (Goodman \& Goodman, 1972 \& 1976; Meyerson et al., 1996).

Complex tasking. Complex tasking encourages both novel and new ways of approaching problems together in a collaborative manner.

Non-routine tasking. Non-routine tasking promotes interdependence as members must rely upon the expertise and experience of others to solve tasks. This promotes idea generation as new ways to solve tasking scenarios are presented.

Time sensitive tasking (i.e., deadlines). People under time pressures make greater use of information processing, emphasizing speed and immediate confirmation rather than evidence-based information processing that is focused on accuracy. A speed-accuracy trade-off is performed. This time sensitive pressure benefits the rapid generation of ideas over a prolonged analysis of consequences. 
Important tasking. Important tasking implies consequences for failure. The level of tasking importance and the perceived level of possible ramifications for failure pushes members into action. The result is an active, proactive and enthusiastic participation by the team members.

Thus far, this paper has addressed team creativity and the collective environment necessary for fostering team creativity. Swift trust, and its properties that promote the collaborative experience leading to team creativity, has also been discussed. However, the moderating effects of the leader-member relationship and history have only been touched upon. In the following two sections, the moderating factors of the leader-member relationship and history are explored.

\section{LEADER - MEMBER RELATIONSHIP (MODERATING FACTOR \#1)}

Literature supports a connection between the leader-member relationship and team creativity. Some theorists have focused on the need for leadership participation (Kanter, 1983) while others have provided lists of specific activities that leaders should engage in to allow creativity to emerge (Amabile, 1988). Scott and Bruce (1994) suggest that the quality of the relationship between the supervisor and subordinates can determine the latitude, influence, and autonomy given to subordinates which influences the creativity and innovation process. Scott and Bruce propose a model of individual innovative behavior. Creative behavior, either pro or con, can be influenced by a leader's expectations, the leader-member exchange or leadership style (Scot \& Bruce, 1994).

Leader - member relationships can influence team creativity and performance. Schaubroeck et al. (2011) associate cognitive and affect-based based trust in the leader with the creative capacity of a team. A member's belief in the competency of the team leader leads to cognitive trust. When the cognitive trust placed in a leader increases, as evidenced through an increase in individual participation, so does team creativity and team innovation. Further, when team members perceive that they are pursuing the same objectives as their leaders, they are apt to perform at higher levels of creativity. Erturk (2012) credits the effects of psychological empowerment and trust in the supervisor to enhancing creativity. The antecedents to improved creativity, guided by the leader, include: (a) as sense of empowerment; (b) a sense of meaning to the task; (c) a perceived competence of the leader; (d) selfdetermination; and, (e) impact - a sense of accomplishment. Since the opposite is true (i.e., a failure to provide the antecedents to improved creativity), the leader-member relationship can have a regulating effect on the collective environment of the team. So, why is this important? Because teams require leadership guidance in order to succeed. As demonstrated from these studies, the leader-member relationship should be cultivated to free the collaborative environment of managerial constraint, empowering its members, so the team is able succeed.

\section{WORKING HISTORY (MODERATING FACTOR \#2)}

The influence of swift-trust on team creativity does not diminish. However, the form of trust, and level of creativity between team members, thickens or thins over time. Within the swift-trust environment, member behavior presupposes trust is already present. As such, a form of cognitive trust is present. Cognitive trust is defined as one's willingness to rely on another team member's expertise and reliability (Barczak, 2010). However, as time passes, a more durable form of trust develops between team members (Robert et al., 2009). Robert et al. (2009) looks upon the formation of team trust and its influences on team activities, including creativity, as being a two stage process. As team members accumulate sufficient information about each other, the effects of swift-trust diminish but, not completely. As history accumulates, cognitive trust deepens and an affect-based form of trust develops. Affect-based trust "is the confidence one places in a team member based on one's feelings of caring and concern illustrated by the co-worker" (Barczak, 2010) over time. Positive associations over time will strengthen the affect-based trust amongst team members while poor associations will have the opposite effect and diminish team trust. As such, the histories between team members can have a regulating effect on the collective environment of the team. Put simply, cognitive trust reflects an objective assessment at any one point in time, while affect-based trust is a subjective appraisal tested over time (Pi, 2012).

Team trust is positively associated with team creativity. Barczak (2010), studies the connections between affect-based and cognitive trust and team creativity. Creative teams, as asserted, are a valuable and necessary tool for organizations because they produce novel ideas and solutions that maintain the firm's competitive advantage. 
When team members trust each other, they are more likely to work closely together and share knowledge to accomplish shared goals. Through a survey of 82 student teams at a large university in the northeast United States, the author tests team creativity as an outcome of a collaborative culture. The author concludes that affect-based trust and cognitive trust are positively associated with the development of a collaborative culture that improves team creativity.

These studies are important because they emphasis the fluid environment of both trust and creativity within teams. Teams that demonstrate team cohesion and team trust are more likely to be tolerant and accepting of divergent ideas and viewpoints (Barczak et al., 2010) thereby, improving the team creativity process.

\section{TEAM ADJUSTMENTS}

Team adjustments may be necessary from time to time to maintain the collaborative team dynamics first established by swift trust. Without periodic adjustments, the effects of swift trust will decline and knowledge-based trust formed using team member behaviors will become dominant (Robert et al., 2009). This suggests that the positive energies created from swift trust are strongest within the temporary environment and adjustments to the antecedents leading to swift trust (i.e., team composition and team tasking) may be required. Such ascertains are bolster by the earlier findings of Goodman and Goodman (1976) and Meyerson et al. (1996) discussed earlier in this paper. As such, adjustment steps might be considered including the replacement or reassignment of its members, changes to tasking assignments and new completion deadlines.

\section{CONCEPTUAL MODEL}

From the literature presented, the development of a conceptual model is presented (see Figure 1, for conceptual model of the team creative process). Based on the literature reviewed, the interrelationships between the various model elements will now be discussed.

Figure 1. Conceptual Model of Team Creativity

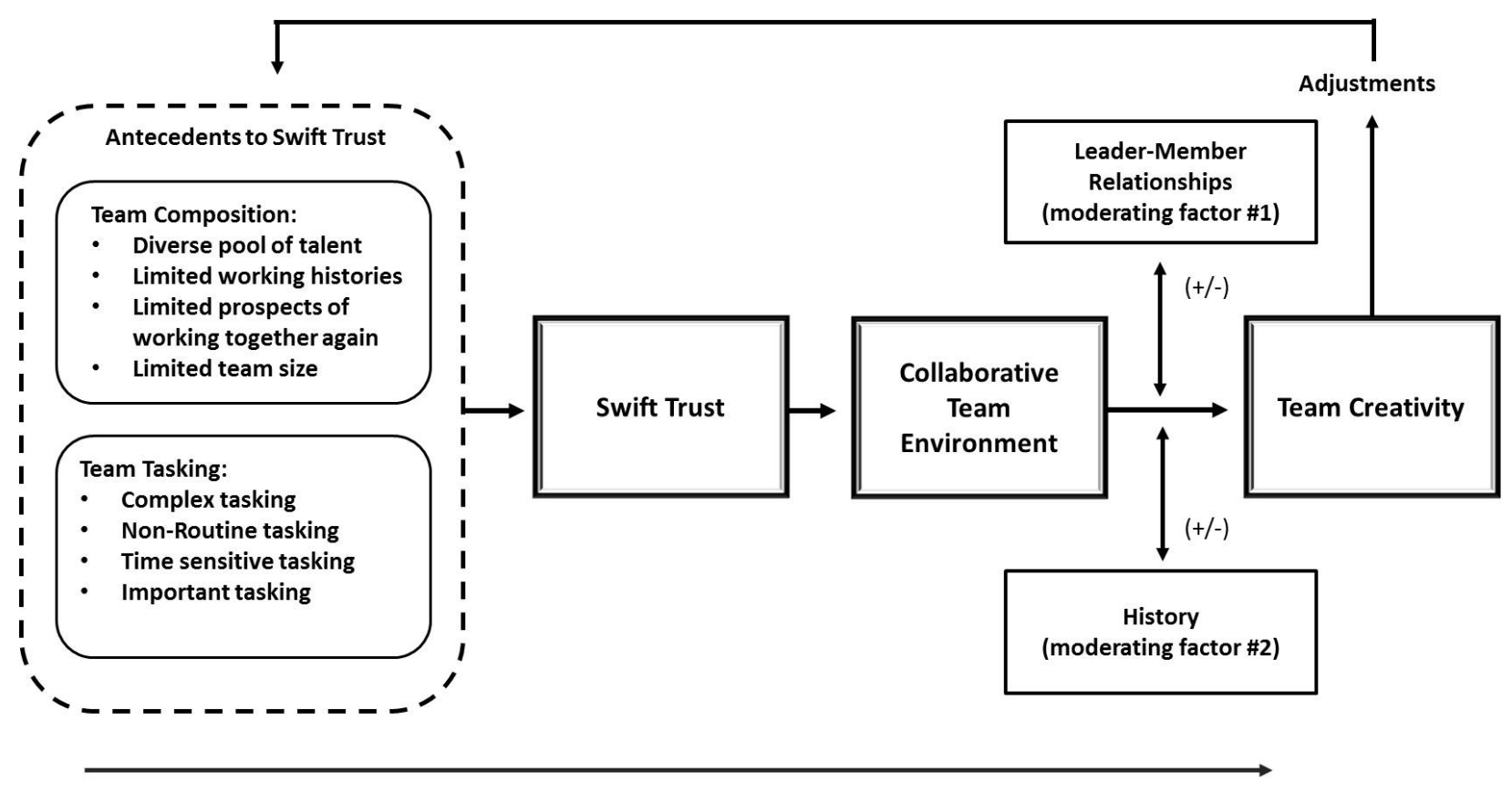




\section{DISCUSSION}

Team creativity begins with the establishment of a swift-trust environment. The swift-trust environment is developed as a consequence of the group composition and tasking motivations provided. Group composition limits the number of members and is planned to provide a diverse pool of talent, composed of members with limited histories of working together or prospects of working together again. Team assignments are complex, non-routing, time-sensitive and important in nature. These precursors to swift trust bring about swift trust, a milieu that presupposes the elements of trust exist and encourages the collective behaviors of its members. This in turn, leads to a proper collaborative team environment which enables team creativity to thrive. History and the lead-member relationships provide moderating affects and can alter the team energies first created by swift trust. Therefore, as time passes, adjustments to the team composition may be required to maintain the creative environment. To illustrate, a practical example is offered.

Northrop Grumman and Lockheed Martin, as an example, design, build, and deploy Unmanned Aerial Vehicles (UAV's). Each corporation has invested heavily to develop core capabilities in this area which will provide them with a competitive advantage. However, in today's competitive environment, companies need to continually innovate in order to survive. Both companies have established Research and Development (R\&D) projects but often establish 'tiger teams' for the sole purpose of generating new ideas for R\&D investment to sustain their competitive advantages. So, how would this work?

Northrop Grumman or Lockheed Martin decides to establish a new R\&D or "tiger" team. A diverse pool of talent is identified and assigned to the task. Talent is chosen to minimize any historical references between team members and they are told it is not likely they will work together again. The team is limited in number. The leadership makes it clear that the task is important, time sensitive and non-routine. The team is then encouraged to do whatever it takes, regardless of prior roles, to work interdependently on the complex task to its final completion. Finally, a supportive supervisor is chosen. Based on literature, a form of collective perception and presupposed trust between members will occur. Ideas will flow as each member wants the team to succeed - the team creativity process will be stimulated and positioned for success through the establishment of a collaborative team environment.

Follow-on tasks, as required to continue the innovation process, may be assigned to the same team as the trust construct begins to shift based on the perceived experiences of the team members. As time progresses, cognitive and affect-based forms of trust either solidifies the team or signals a dismantling or change in the team may be necessary. If managed correctly, the expertise and working rapport developed during the initial and critical start-up phase (swift-trust) will continue. However, the presumption of trust seen during the initial phases of team development will be replaced by the observed competence and affection developed between the team members. Supervisor-Member relationships and history will encourage creative thinking through competence, empowerment, task meaning and task impact. This scenario fits well with the principles of swift-trust and team creativity described above. Emerging from these inputs will be a collaborative team environment that leads to team creativity.

\section{MANAGEMENT IMPLICATIONS}

This paper contributes to the discussion of team creativity by introducing the concepts of swift-trust early in the team formation process. In using swift-trust, creativity, the first step in the innovative process, is started without delay. As time progress, a sustaining form of trust and enhanced creativity develops leading to team creativity. The positive impacts of both cognitive and affect-based trust suggest that managers need to recognize the importance of team member perceptions on the reliability and competence of their colleagues. To aid these perceptions, the selection of team members and associated competencies becomes critical to team success. Additionally, this paper illustrates the importance of leader-member trust. The choice of leadership and his or her association with team members can pro-actively benefit or hinder team creativity.

\section{LIMITATIONS AND FUTURE RESEARCH}

This paper proposes a team creativity model based upon the initializing concept of swift-trust. The model reflects the findings of literature as it pertains to building team creativity. However, this paper also has limitations 
and provides opportunities for further research. First, the topic of innovation, the subsequent step to creativity, is not fully addressed. This presents an opportunity for further expansion of the model as presented. Second, the concept of swift-trust, as it relates to creativity, lacks a diversity of empirical testing. It is suggested that further empirical studies are warranted to validate the model as presented this paper.

\section{CONCLUSION}

The primary goal of this paper is to answer a central question: does swift-trust stimulate the team creativity process? Most literature pertaining to swift-trust and creativity focuses on the individual within the construct of a temporary systems environment. Most literature on creativity emphasizes the pro-active motivational state of the individual while team creativity is generally discussed as an outcome. Trust and knowledge sharing have been implicated in the creation of a collaborative environment that leads to creativity. Yet, little attention has been placed on the early stimulation of team creativity through the careful manipulation of the team construct with swift-trust.

In today's competitive environment, companies need to continually innovate in order to survive. Creativity, the first step in innovation, can be stimulated by swift-trust. By building on the works of Goodman and Goodman (1972 \& 1976), Meyerson et al. (1996) and others, a clearer picture of the connection between trust and creativity has been drawn. The formation of a collaborative environment through swift-trust can be quickly accomplished. The works of Robert et al. (2009) and Barczak (2010) expand the proposition of early trust and creativity development by suggesting the concepts of trust and creativity are fluid and can be strengthened or weakened over time. History and leader-member relationships can affect the development of the collaborative environment leading to team creativity. Overall, however, results confirm a proposition that swift trust leads to the rapid establishment of a collaborative team environment which leads to team creativity.

\section{AUTHOR INFORMAITON}

John Berthold, scholar-practioner, is a business executive with $30+$ years of domestic and international management experience, specializing in new business strategy, contract capture and strategy implementation. Dr. Berthold's research interest focuses on trust development and sustainment within groups as influenced by the group leader. John leverages his expertise to lead teams, win contracts, facilitate strategy and teach competitive intelligence. John is retired from Northrop Gumman Corporation and is currently VP of Technical Services for the Sunhillo Corporation. His B.A. is from the George Washington University, M.B.A. from Colorado State University and Doctor of Management from University of Maryland University College.

\section{REFERENCES}

Amabile, T. (1996). Creativity and innovation in organizations. Harvard Business School, 1-15.

Atkinson, S., \& Butcher, D. (2003). Trust in managerial relationships. Journal of Managerial Psychology, 282-304. doi: 10,1108/02683940310473064

Barczak, G., Lassk, F., \& Mulki, J. (2010). Antecedents of team creativity: An examination of team emotional intelligence, team trust and collaborative culture. Creativity and Innovation Management, 19(4), 332- 345.

Chen, M. (2007). Entrepreneurial leadership and new ventures: Creativity in entrepreneurial teams. Creativity and Innovation Management, 16(3), 239-249.

Chen, G., Farh, J., Campbell-Bush, E., \& Wu, X. (2013). Teams as innovative systems: Multivational antecedents of innovation in R\&D teams. Journal of Applied Psychology, 1-10.

Erturk, A. (2012). Linking psychological empowerment to innovation capability: Investigating the moderating effect of supervisory trust. International Journal of Business and Social Science, 3(14), 153-165.

Goodman, A., \& Goodman, L. (1976). Some management issues in temporary systems: A study of professional development and manpower - the theater case. Administrative Science Quarterly, 494-501.

Goodman, A., \& Goodman, L. (1972). Theater as a temporary system. California Management Review, 103-108.

Kramer, R. (1999). Trust and distrust in organizations: Emerging perspectives, enduring questions. Annual Review of Psychology, 569-98.

Kramer, R. (2010). Collective trust within organizations: Conceptual foundations and empirical insights. Corporate Reputation Review, 13, 82-98. 
Myerson, D., Weick, K., \& Kramer. (1996). Swift trust and temporary groups. From the book by Kramer, R., \& Tyler, T. (1996), Chapter 9. Trust in organizations: Frontiers of theory and research. $166-195$. Thousand Oaks, CA: SAGE Publications, Inc.

Paulus, P. (2000). Groups, teams, and creativity: The creative potential of idea-generating groups. Applied Psychology, 49(2), 237-262.

Pi, S, Liao, H., \& Chen, H. (2012). Factor that affect consumers trust and continuous adaption of online financial services. International Journal of Business and Management, 7(9), 108-119.

Robert, L., Dennis, A., \& Hung, Y. (2009). Individual swift trust and knowledge-based trust in face-to-face and virtual team members. Journal of Management Information Systems, 26, 241 - 279. doi: 10.2753/MIS0742-1222260210

Schaubroeck, J., Lam, S., \& Peng, A. (2011). Cognition-based and affect-based trust as mediators of leader behavior influences on team performance. American Psychological Association, 96(4), 863-871.

Schein, E., H. (2004). Organizational Culture and Leadership ( $3^{\text {rd }}$ ed.). San Francisco, CA: Jossey-Bass.

Scott, S., \& Bruce, R. (1994). Determinants of innovative behavior: A path model of individual innovation in the workplace. Academy of Management Journal, 37(3), 580-607.

Tyler, T., \& Kramer, R. (1996). Whither Trust?. From the book by Kramer, R., \& Tyler, T. (1996), Chapter 9. Trust in organizations: Frontiers of theory and research. 1-15. Thousand Oaks, CA: SAGE Publications, Inc.

West, M. (2002). Sparkling fountains or stagnant ponds: An integrative model of creativity and innovation implementation in work groups. Applied Psychology, 51(3), 355-424.

Zhang, A., Tsui, A., Jiwen, S., Li, C., \& Jia, L. (2008). How do I trust thee? The employee-organization relationship, supervisory support, and middle manger trust in the organization. Human Resources Management, 47, 111132. doi: $10.1002 / \mathrm{hrm} .20200$ 


\section{NOTES}

